\title{
ON P-SOLUBLE RINGS
}

\author{
BY \\ JAKOB LEVITZKI
}

1. Introduction. The present paper is the continuation of a recent study [10](1) of algebraic algebras and more generally of I-rings and FI-rings, i.e. rings with nonzero idempotents in every non nil r. ideal (= right ideal), respectively rings whose homomorphic images are I-rings. The aim is to extend certain structural results on $\mathrm{I}_{1}$-rings and $\mathrm{I}_{2}$-rings (i.e. I-rings with bounded index, respectively those whose primitive images are of bounded index) which were considered in [10] to a much wider class of I-rings by abolishing the restriction that the index should be bounded, even for the primitive images. This restriction, which in the case of $\mathrm{I}_{2}$-rings is equivalent to the minimal condition for the $r$. ideals of every primitive image, is replaced by a weak chain condition, the so-called D-condition (see Definition 6.2) which is imposed on a certain class of the principal $r$. ideals of every primitive image. Thus, e.g. I-rings whose primitive images coincide with their socle or, more generally, possess a composition chain of iterated socles, fall under the new category.

In the structure theory of $I_{1}$-rings and $I_{2}$-rings a fundamental role was played by the so-called matrix ideals, that is, ideals with central idempotent, isomorphic with a total matrix ring of finite degree over a plain ring. For the type of rings considered here matrix ideals are no longer available. In general the center of these rings, even if they are semi-simple, is vacuous (i.e. $=0$ ). We introduce a class of $r$. ideals, the so-called r. P-ideals which play here a similar role to that of the matrix ideals in the former theory. A r. ideal is a r. P-ideal if it is a plain ring modulo its radical. The sum $S^{\prime}$ of all $r$. P-ideals of a ring $\subseteq$ is called the P-socle of $\subseteq$. This is an ideal that contains also all 1. P-ideals of the ring. We show e.g. that every FI-ring satisfying the $\mathrm{D}$-condition for its primitive images is $\mathrm{P}$-soluble, i.e. it possesses a composition chain of ideals $\left\{A_{\sigma}\right\}$ such that for every $\sigma$ the ring $A_{\sigma+1}$ $-A_{\sigma}$ is the $\mathrm{P}$-socle of $S-A_{\sigma}$. Conversely, the primitive images of a $\mathrm{P}$-soluble FI-ring satisfy the D-condition. It follows that an FI-ring is $\mathrm{P}$-soluble if and only if every primitive image is $\mathrm{P}$-soluble. The $\mathrm{D}$-condition for the primitive images of an I-ring implies its validity for the ring itself. In the case of algebraic algebras we are able to show that the converse is also true.

In $\S 8$ we apply these results to obtain a generalization of Kaplansky's theorems concerning Kurosch's problem (compare [6], [7], and [10]). In handling this problem, the locally finite kernel which was introduced in [10]

Presented to the Society, September 5, 1952; received by the editors September 6, 1952 and, in revised form, June 1, 1953.

(1) Numbers in brackets refer to the bibliography at the end of the paper. 
again proved to be a rather useful tool. Another application is discussed in $\$ 7$, where a result due to $\mathrm{R}$. Baer [2] concerning rings that possess a composition chain of iterated socles is sharpened and generalized.

In order to avoid repetitions in proving a certain category of theorems, or formulating definitions of similar character for the various types of rings that are considered here, it was found to be expedient to present such material in a unified manner for a class of rings of a more general type, the socalled (E-rings ( $\$ 2)$. This section is intimately connected with recent investigations of A. S. Amitsur [1] who has undertaken an axiomatic study of certain properties of rings and other more general algebraic domains. However, in our $\S 2$, which is of a preparatory character, no attempt was made to achieve the greatest possible generality.

As to terminology, compare [10]. In this paper "ideal" always means two-sided ideal.

2. A general notion of solubility. Denote by $\&$ a ring property satisfying the conditions 2.1-2.5 specified below. A ring, a r. ideal or a 1. ideal having property $\mathbb{E}$ will be referred to as an (E-ring, a r. (E-ideal and a 1. E-ideal respectively.

Condition 2.1. Every r. ideal and every 1 . ideal of an $\mathbb{E}$-ring are $\&$-rings.

Condition 2.2. If $A$ is a r. $\leftleftarrows$-ideal of a ring $S$ and $s \in S$, then $s A$ is a r. E-ideal. Similarly for 1 . E-ideals.

Conditron 2.3. Every ring $S$ such that $S^{2}=0$ is an E-ring.

Condition 2.4. If $A$ is an ideal in $S$ such that $A^{2}=0$, then $S-A$ is an (E-ring if and only if $S$ is an (E-ring.

Condition 2.5. Every l. ideal generated by an element of a r. ( -ideal is a 1. (E-ideal (and similarly, by interchanging r. ideals and 1. ideals).

If every homomorphic image of an \&-ring $S$ is an E-ring, we say that $S$ has the FE-property. A ring, a r. ideal or a 1. ideal having the property F\& will be referred to as an F\&-ring, r. FE-ideal or 1. FE-ideal respectively.

THEOREM 2.1. Every r. ideal $R$ of an FE-ring $S$ is an F\&-ring.

Proof. Let $A^{\prime}$ be an ideal in $R$. We have to show that $R-A^{\prime}$ is an (E-ring. Put $A^{\prime \prime}=A^{\prime} R$ and $A=S A^{\prime \prime}+A^{\prime \prime}$. Then $A$ is an ideal in $S, R A \subseteq A^{\prime \prime}$ and we have

$$
\begin{aligned}
R-A^{\prime} & \cong\left(R-A^{\prime \prime}\right)-\left(A^{\prime}-A^{\prime \prime}\right) \\
\left(R-A^{\prime \prime}\right) & \cong(R-R A)-\left(A^{\prime \prime}-R A\right) .
\end{aligned}
$$

Since evidently $A^{\prime 2} \subseteq A^{\prime \prime}, A^{\prime \prime} \subseteq R A$, it follows by condition 2.4 that $R-A^{\prime}$ is an E-ring whenever $R-R A$ is an \&-ring. Now

$$
(R-R A)-[(R \cap A)-R A] \cong R-(A \cap R) .
$$

For the ring $B=(R \cap A)-R A$ we evidently have $B^{2}=0$, and thus by condition 2.4 it remains to show that $R-(A \cap R)$ is an Eै-ring. In fact 


$$
R-(A \cap R) \cong(R+A)-A
$$

and since $S$ is an FE-ring we know that $S-A$ is an $\mathbb{E}$-ring. Hence by condition 2.1 also the r. ideal $(R+A)-A$ and hence $R-(A \cap R)$ is an (F-ring, q.e.d.

Definition 2.1. The sum of all r. E-ideals of a ring $S$ is called the $\mathbb{E}$-socle of $S$.

THEOREM 2.2. The (5-socle $S^{\prime}$ of a ring $S$ is an ideal which may be obtained also as the sum of all $l$. (5-deals of $S$.

Proof. If $a \in S^{\prime}$, there exists a finite set of r. (F-ideals $R_{1}, R_{2}, \cdots, R_{n}$ such that $a=\sum_{i=1}^{n} r_{i}, r_{i} \in R_{i}$. Then $s a=\sum_{i=1}^{n} s r_{i} \in \sum s R_{i}$. By condition 2.2 the $s R_{i}$ are also r. (E-ideals, hence $s a \in S^{\prime}$, which shows that $S^{\prime}$ is two-sided. If further $L$ is any 1 . ( 5 -ideal and $a \in L$, then by condition 2.5 we know that the $\mathrm{r}$. ideal $(a)_{r}$ generated by $a$ is a r. ( -ideal. Hence $L$ lies in a sum of r. (Fideals, and thus for the sum $S^{\prime \prime}$ of 1 . (E-ideals we have $S^{\prime \prime} \subseteq S^{\prime}$. Similarly $S^{\prime} \subseteq S^{\prime \prime}$. Hence $S^{\prime \prime}=S^{\prime}$, q.e.d.

Definition 2.2. Let $S^{\prime}$ be the $\left(\right.$-socle of a ring $S$. If $S-S^{\prime}$ is a nil-ring, $S$ is called $\leftleftarrows$-reducible. A ring whose homomorphic images are $\mathbb{E}$-reducible is called FE-reducible.

One verifies readily the following

LEMмA 2.1. If a r. ideal $R$ of a ring $S$ is an (5-reducible ring, then also $R S$ is (5-reducible. If $R^{\prime}$ is the (5-socle of $R$, then $R S-R^{\prime} S$ is nil and $R^{\prime} S$ is a subset of the (E-socle of $R S$.

With the help of Lemma 2.1 and Theorem 2.1 one readily obtains

THEOREM 2.3. The property of (E-reducibility (F\&-reducibility) is an (E-property (FF-property) $\left({ }^{2}\right)$.

Definition 2.3. A well ordered ascending chain (not necessarily properly ascending) of ideals $\left\{A_{\sigma}\right\}$ in a ring $S$, beginning at $A_{0}=0$ and terminating at $A_{\tau}$, is called a composition chain if for every limit ordinal $\lambda \leqq \tau$ we have $A_{\lambda}=\mathrm{U}_{\rho<\lambda} A_{\rho}$. The ordinal $\tau$ is called the length of the chain. If $A_{\tau}=S$ we say that $S$ has the composition chain $\left\{A_{\sigma}\right\}$.

Lemma 2.2. Denote by $\left\{A_{\sigma}\right\}$ a composition chain for $S$. Then: (a) Let $T$ be a subring of $S$ and put $B_{\sigma}=T \cap A_{\sigma}$. Then $\left\{B_{\sigma}\right\}$ is a composition chain for $T$, and $B_{\sigma+1}-B_{\sigma}$ is isomorphic with a subring of $A_{\sigma+1}-A_{\sigma}$. (b) Let $T$ be an ideal in $S$ and put $B_{\sigma}=\left(T+A_{\sigma}\right)-T$. Then $\left\{B_{\sigma}\right\}$ constitutes a composition chain for $S-T$, and $B_{\sigma+1}-B_{\sigma}$ is a homomorphic image of $A_{\sigma+1}-A_{\sigma}$.

Proof. Follows easily by standard argument.

Definition 2.4. If a ring $S$ has a composition chain $\left\{A_{\sigma}\right\}$ such that

(2) Nillity is an F(E-property. Theorem 2.3 remains valid if in Definition 2.2 we replace nillity by any $F$ \&-property. 
$A_{\sigma+1}-A_{\sigma}$ is $\mathbb{E}$-reducible, then $S$ is called $\mathbb{E}$-soluble. If all homomorphic images of $S$ are $\mathbb{E}$-soluble then $S$ is called FE-soluble.

TheOREM 2.4. (a) Every r. ideal of an (E-soluble ring is (E-soluble. (b) If $\left\{A_{\sigma}\right\}$ is an (E-chain for a ring $S$ such that $A_{\sigma+1}-A_{\sigma}$ is FE-reducible, then $S$ as well as every $r$. ideal in $S$ is FE-soluble. (c) Every nonzero $r$. ideal of an (E-soluble (Fङ-soluble) ring contains a nonzero r. (E-ideal (r. Fङ-ideal) of $S$.

Proof. (a) By Theorem 2.3 every r. ideal in an \&-reducible ring is also (5-reducible. Hence if in Lemma 2.2 we identify the ring $T$ with a r. ideal $R$, part (a) of our theorem becomes a consequence of part (a) of Lemma 2.2. Part (b) is a consequence of Theorem 2.1 and Lemma 2.2, while (c) results with the help of Lemma 2.1.

TheOREM 2.5. A ring $S$ is FE-soluble if and only if every nonzero homomorphic image of $S$ contains a nonzero $r$. F\&-ideal.

Proof. The "only if" part of the theorem is contained in part (c) of Theorem 2.4. To prove the "if" part, consider the sum $A_{1}$ of all r. F\&-ideals of the ring. If $S \neq 0$ then $A_{1} \neq 0$ and by Theorem 2.3 it follows readily that $A_{1}$ is an FE-reducible ring and an ideal in $S$. If $S=A_{1}$ the theorem is proved. If not then $S-A_{1}$ contains nonzero $r$. FE-ideals and the process can be continued. The familiar use of transfinite induction $\left(^{3}\right)$ leads to an FE-chain for $S$.

3. Nil-ideals and I-rings. In the present section we collect some simple facts concerning nil-ideals and I-rings which will be needed later. It is readily verified that an I-ring (a nil-ring) is a special type of an (E-ring (FE-ring). We denote by $N(S)$ the nil-radical of a ring $S$, i.e. the maximal nil-ideal of $S$ and by $N^{*}(S)$ the sum of all r. nil-ideals of $S$. By Theorem 2.2 we know that $N^{*}(S)$ is an ideal that contains also all 1 . nil-ideals of the ring. We recall that it is still an open question whether or not $N^{*}(S)=N(S)$. One verifies readily the following

LEMma 3.1. All r. nil-ideals of a ring $S$ that are contained in a $r$. ideal $R$ of $S$ belong to $N(S)$ if and only if the following condition holds:

$$
N^{*}(R) \cdot R \subseteq N(S) .
$$

Proof. Suppose that (4) holds and let $N_{1}$ be any r. nil-ideal of $S$ such that $N_{1} \subseteq R$. Then by $N_{1} \subseteq N^{*}(R)$ and $N_{1} \subseteq R$ it follows in view of (4) that $N_{1}^{2}$ $\subseteq N_{1} R \subseteq N(S)$, i.e. $N_{1}$ is a nilpotent r. ideal modulo $S-N(S)$. Since $S-N(S)$ has no nilpotent ideals, we must have $N_{1} \subseteq N(S)$. Conversely, suppose that every r. nil-ideal of $S$ that lies in $R$ belongs to $N(S)$, and let $N^{\prime}$ be any r. nilideal of $R$. Then $N^{\prime} R$ is a r. nil-ideal of $S$ that lies in $R$. Hence $N^{\prime} R \subseteq N(S)$, which shows that (4) is valid.

REMARK. It follows easily that whenever (4) holds we have also

(3) Compare e.g. [2, Theorem 5.1]. 


$$
N(R)=N^{*}(R) .
$$

Indeed, in this case $N^{*}(R)$ is a nil-ring and thus it must coincide with the maximal nil-ideal of $R$.

LEMma 3.2. If (4) holds for a $r$. ideal $R$ of a ring $S$, then it holds also for every r. ideal $s R, s \in S$.

Proof. Suppose that (4) is not valid for some $s R$. This means that for some r. nil-ideal $s R_{1}$ of $s R$, where $R_{1} \subseteq R$, we have $s R_{1} \cdot s R \nsubseteq N(S)$. Hence $\left(s R_{1} \cdot s R\right)^{3}$ $\Phi N(S)$. Thus there is a triple of elements $s r_{i}, r_{i} \in R_{1}, i=1,2,3$, such that $s r_{1} \cdot s r_{2} \cdot s r_{3} \notin N(S)$. Put $a=s r_{2} \cdot s r_{3}$, then $s r_{1} \cdot a \notin N(S)$. However, $a S \subseteq s R_{1}$, i.e. $a S$ is nil. Hence also $r_{1} a S$ is nil. Since $r_{1} \cdot a S \subseteq R$, this implies by (4) that $r_{1} a S \subseteq N(S)$. Hence also $s r_{1} a S \subseteq N(S)$, i.e. $s r_{1} \cdot a \in N(S)$-a contradiction.

Lemma 3.3. If for an arbitrary set of $r$. ideals $\left\{R_{i}\right\}$ the relation (4) holds, then it holds also for $R=\sum R_{i}$.

Proof. If not, then $R$ contains a r. nil-ideal $R^{\prime} \Phi N(S)$. Then $R^{\prime 2} \subseteq R^{\prime} R$ $\Phi N(S)$. Hence for some $R_{i}$ we must have $R^{\prime} R_{i} \Phi N(S)$. For some $a \in R^{\prime}$ we get $a^{\prime} R_{i} \nsubseteq N(S)$. However $a^{\prime} R_{i}$ is nil and since (4) holds for $R_{i}$, by Lemma 3.2 it holds also for $a^{\prime} R_{i}$, which implies $a^{\prime} R_{i} \subseteq N(S)$-a contradiction.

LEMma 3.4. If (4) holds for a $r$. ideal $R$ of $S$, then it holds also for the ideal $A=S R+R$.

Proof. By Lemma 3.2 we have $N^{*}(s R) s R \subseteq N(S)$ for every $s \in S$, which by Lemma 3.3 yields $N^{*}(S R+R) \cdot(S R+R) \subseteq N(S)$.

Lemma 3.5. If for an ideal $A$ of a ring $S$ we have $N^{*}(A)=N(A)$ and $N^{*}(S-A)=N(S-A)$, then $N(S)=N^{*}(S)$.

Proof. First note that for an ideal $A$ we have $N(A) \subseteq N(S)$. If now $R$ is a r. nil-ideal in $S$, then $(R+A)-A$ is nil in $S-A$. By $N^{*}(S-A)=N(S-A)$ this implies that $(S R+R+A)-A \subseteq N(S-A)$. Now

$$
(S R+R+A)-A \cong(S R+R)-[A \cap(S R+R)] .
$$

Since $R$ is nil, also $R A$ is nil, which by $R A \subseteq A$ implies $R A \subseteq N^{*}(A)=N(A)$ $\subseteq N(S)$. Thus $(S R+R) A \subseteq N(S)$. Since $[(S R+R) \cap A]^{2} \subseteq(S R+R) A \subseteq N(S)$ we have $(S R+R) \cap A \subseteq N(S)$, which by (6) implies that $S R+R$ is nil, i.e. $S R+R \subseteq N(S)$. Hence every r. nil-ideal lies in $N(S)$, i.e. $N^{*}(S)=N(S)$.

Lemma 3.6. If a ring $S$ contains a set of $r$. ideals $\left\{R_{i}\right\}$ such that $A=\sum R_{i}$ is an ideal, $S-A$ is nil and $R_{i}$ satisfies (4), then $N^{*}(S)=N(S)$.

Proof. This is a consequence of Lemmas 3.3 and 3.5.

Lemma 3.7. If for a composition chain of ideals $\left\{A_{\sigma}\right\}$ terminating at $A_{\tau}$ we have $N^{*}\left(A_{\sigma+1}-A_{\sigma}\right)=N\left(A_{\sigma+1}-A_{\sigma}\right)$ for $\sigma+1 \leqq \tau$, then $N^{*}\left(A_{\tau}\right)=N\left(A_{\tau}\right)$. 
Proof. This follows readily with the help of Lemma 3.5.

Lemma 3.8. If for a set of $r$. I-ideals (FI-ideals) $\left\{R_{i}\right\}$ condition (4) holds for every $r$. ideal of the set (respectively for every image of $R_{i}$, induced by any homomorphism of $S$ ), then $A=\sum R_{i}$ is also a r. I-ideal (FI-ideal).

Proof. Let $R$ be a non-nil r.ideal in $A$ and $b \in R$ a non nilpotent element. For some integer $n$ we have $b \in \sum_{i=1}^{n} R_{i}$. Then $\sum_{i=1}^{n} b R_{i}$ is not nil. Since by Lemma 3.2 condition (4) holds for every $b R_{i}$, it follows that for some $i$ the r. ideal $b R_{i}$ is not nil. Since with $R_{i}$ also $b R_{i}$ is a r. I-ideal $\left({ }^{4}\right)$, it follows that $b R_{i}$ contains a nonzero idempotent $e$, and we have $e \in R$, i.e. $A$ is an I-ring. The remaining part of the theorem (for FI-rings) is evident.

REMARK. We may say that a property $\&$ is additive for a ring $S$ if the sum of two r. (E-ideals of $S$ is again a r. E-ideal. With the help of Lemma 3.8 it can be readily deduced that the I-property is an additive E-property for a ring $S$ if and only if nillity is an additive $E$-property for $S$.

One verifies readily that if for an ideal $A$ of a ring $S$ both $A$ and $S-A$ are I-rings (FI-rings), then also $S$ itself is an I-ring (FI-ring). If further $S$ is the union of an ascending chain of ideals each of which is an I-ring (FI-ring), then also $S$ is an I-ring (FI-ring). These remarks in conjunction with Lemmas 3.6-3.8 may be summarized in the following two theorems:

Theorem 3.1. (a) If every $r$. (E-ideal $R$ of an (E-reducible ring $S$ satisfies condition (4), then $N^{*}(S)=N(S)$. (b) If $S$ is an (E-soluble ring and $\left\{A_{\sigma}\right\}$ is an (E-chain for $S$ such that for any $\sigma$ every $r$. (E-ideal of $A_{\sigma+1}-A_{\sigma}$ satisfies condition (4), then $N^{*}(S)=N(S)$.

THEOREM 3.2. (a) If for $a$ set of $r$. I-ideals (FI-ideals) $\left\{R_{i}\right\}$ of a ring $S$ condition (4) holds for every $R_{i}$ (respectively for every image of $R_{i}$ induced by any homomorphism of $S$ ), then the sum $A=\sum R_{i}$ is also a $r$. I-ideal (FI-ideal). (b) If $\left\{A_{\sigma}\right\}$ is a composition chain of ideals for a ring $S$ such that $A_{\sigma+1}-A_{\sigma}$ is an I-ring (FI-ring) for every $\sigma$, then also $S$ is an I-ring (FI-ring).

For later reference we mention here a case where condition (4) is automatically invariant under every homomorphism of $S$. First note that

LEMma 3.9. If for a ring $T$ the difference ring $T-N(T)$ is strongly regular, then in every homomorphism $T \sim T^{\prime}$ the image $N^{\prime}(T)$ of $N(T)$ is $=N\left(T^{\prime}\right)$.

Proof. If $A$ is the kernel of the homomorphism, then $B=(T-A)$ $-[(N(T)+A)-A] \cong T-[N(T)+A] \cong[T-N(T)]-[(N(T)+A)-N(T)]$, which shows that $B$ is a homomorphic image of the strongly regular ring $T-N(T)$. Hence $B$ itself is strongly regular, which by the nillity of $[N(T)+A]$ $-A$ implies that $N(T-A)=(N(T)+A)-A \cong N^{\prime}(T)$.

This lemma yields the following sharper formulation of Theorem 3.2(a) in the strongly regular case.

(4) We have remarked already that I-rings are special E-rings. 
THEOREM 3.3. If for a set of $r$. FI-ideals $\left\{R_{i}\right\}$ condition (4) holds for every $R_{i}$ and $R_{i}-N\left(R_{i}\right)$ is strongly regular, then $A=\sum R_{i}$ is an FI-ring.

4. On P-soluble rings. We now single out a class of $\&$-rings, the so-called $P$-rings, which are fundamental for the following considerations.

Definition 4.1. A ring $T$ is called a P-ring if $T-N(T)$ has no nonzero nilpotent elements. An equivalent definition is: The nilpotent elements of $T$ form an ideal. A P-ring which is a r. ideal in a ring $S$ will be referred to as a r. P-ideal. Similarly 1. P-ideals are defined. A ring whose homomorphic images are $\mathrm{P}$-rings is called an FP-ring.

The following lemmas show that the $\mathrm{P}$-property is a special type of (5-property. First note that if $U$ is a subring of a P-ring $T$, then evidently $N(U) \subseteq N(T)$. Hence

\section{Lemma 4.1. Every subring of a P-ring is a P-ring.}

We further have

Lemma 4.2. If $R$ is a $r$. P-ideal in a ring $S$, then for every $s \in S$ also $s R$ is a r. P-ideal.

Proof. If $a \in s R$ is nilpotent, $a=s r, r \in R$, then also $r s$ is nilpotent. By $r s \in R$ this implies that $r s R$ is nil. Hence $a s R$ and consequently also $s R a$ are nil. Assume further that $s r_{i}, r_{i} \in R, i=1,2$, is a pair of nilpotent elements. Then also $r_{i} s, i=1,2$, are nilpotent. Since $r_{i} \in R$ this implies that also $\left(r_{1}-r_{2}\right) s$ is nilpotent, which in turn shows that $s r_{1}-s r_{2}$ is nilpotent. We have shown that the nilpotent elements of $s R$ form an ideal in $s R$, q.e.d.

LEMMA 4.3. Every $l$.ideal generated by an element of a r. P-ideal is a l. P-ideal.

Proof. The proof is similar to that of Lemma 4.2 and may be omitted.

By definition every nil-ring is a P-ring. One verifies readily that if $A$ is a nil-ideal in a ring $S$, then $S-A$ is a $\mathrm{P}$-ring if and only if $S$ is a $\mathrm{P}$-ring. This, in conjunction with Lemmas 4.1,4.2 and 4.3 shows that any $\mathrm{P}$-ring has the $\&$-ring properties, and $\$ 2$ is now available for P-rings. It may suffice to mention here explicitly that one defines the $\mathrm{P}$-socle of a ring as the sum of all r. P-ideals and that this is an ideal that contains all 1 . P-ideals of the ring. It is clear how P-reducibility (FP-reducibility) and P-solubility (FPsolubility) are to be defined.

5. On P-soluble I-rings. A homomorphic image of an I-ring, provided that the kernel of the homomorphism is nil, is again an I-ring. Since further any I-ring has (E-ring properties, every r. ideal of an I-ring (FI-ring) is also an I-ring (FI-ring). In view of the fact that an FI-ring is plain if and only if it is strongly regular (see [10, Theorem 5.5$]$ ), this leads to

Theorem 5.1. If $R$ is a r. P-ideal in an I-ring (FI-ring), then $R-N(R)$ is plain (strongly regular). 
Corollary. $A r$. P-ideal of an FI-ring is a $r$. FP-ideal $\left({ }^{(5)}\right.$.

The following theorem is to some extent the converse of Theorem 5.1.

Theorem 5.2. If $T$ is a subring of a ring $S$ such that $T-N(T)$ is plain, $N(T) \subseteq N(S)$ and $T S T \subseteq T$, then $R=T+T S$ is a r. P-ideal in $S$.

Proof. Let $b \in R, b \notin N(R)$. Then $b R \Phi N(R)$, hence $b T \Phi N(S)$. Thus $b T$ $\Phi N(S) \cap T=N(T)$ i.e. $b T \Phi N(T)$. Since $b T \subseteq T$ and with $T-N(T)$ also $T$ is an $I$-ring, there is an idempotent $e \neq 0$ such that $e=b t$, where $t=t e \in T$. Denote by $e^{\prime}, b^{\prime}$, and $t^{\prime}$ the images of $e, b$, and $t$ under the natural homomorphism $T \sim \rightarrow T-N(T)$. Then $e^{\prime}=b^{\prime} t^{\prime}, t^{\prime}=t^{\prime} e^{\prime}$. Since $T-N(T)$ is plain, the nonzero idempotent $e^{\prime}$ is in the center [10, Lemma 2.2] and thus $e^{\prime}=b^{\prime} e^{\prime} t^{\prime}$ $=b^{\prime 2} t^{\prime 2}=\cdots=b^{\prime k} t^{\prime k}$ for every $k$. Hence $b^{\prime}$ is not nilpotent, which implies that also $b$ is not nilpotent. Thus we have shown that $N(R)$ contains all the nilpotent elements of $R$, i.e. $R$ is a r. P-ideal.

Definition 5.1. An idempotent $e$ of a ring $S$ is called a P-idempotent if the ring $e S e$ is a P-ring.

Since for an idempotent $e$ we have $N(e S e) \subseteq N(S)$, Theorem 5.2 yields

TheOREM 5.3. If $e$ is an idempotent of an I-ring $S$, then eS is a r. P-ideal if and only if $e$ is a P-idempotent.

The radical (in the sense of Jacobson-Chevalley) of an I-ring $S$ coincides with its nil-radical $N(S)$. Since by definition every r. nil-ideal is a r. P-ideal, the P-socle $S^{\prime}$ of an I-ring contains its radical $N(S)$. Since further every matrix ideal of degree $n$ (see Definition 2.1 in [10]) is a sum of $n$ r. P-ideals $\left({ }^{6}\right)$, it follows that $S^{\prime}$ contains also all ideals whose images modulo $N(S)$ are matrix ideals. Thus for an $\mathrm{I}_{1}$-ring or an $\mathrm{I}_{2}$-ring $S$ such that $S \supset N(S)$, the radical is a proper subset of the $\mathrm{P}$-socle. Of a more general character is the following result.

THEOREM 5.4. If an I-ring $S$ contains an idempotent $e \neq 0$ such that either the ring $T=e S e$ is of bounded index, or every primitive image of $T$ is of bounded index, then the radical $N(S)$ of $S$ is a proper subset of the P-socle $S^{\prime}$.

Proof. By [10, Theorems 3.3 and 3.4] the I-ring $\bar{T}=T-N(T)$ contains nonzero matrix ideals. Hence $\bar{T}$ contains a nonzero P-idempotent $\bar{e}$. Let $e$ be an idempotent that maps into $\bar{e}$ under the natural homomorphism $T \sim \vec{T}$. Then $e$ is a nonzero P-idempotent in $T$. By Theorem 5.3 we know that $e S$ is a r. P-ideal of $S$. Hence $S^{\prime} \supset N(S)$, q.e.d.

(5) In accordance with $\S 2$ a ring $S$ is an FP-ring if every homomorphic image of $S$ is a P-ring.

(6) This is in analogy to the decomposition of a total matrix ring over a division ring into a direct sum of minimal $r$. ideals. 
TheOREM 5.5. If $S$ is a primitive I-ring, then a $r$. ideal $R \neq 0$ in $S$ is a minimal $r$. ideal if and only if it is a r. P-ideal.

Proof. Suppose that $R$ is a r. P-ideal in $S$. Since $S$ is primitive, $R$ is not nil and thus contains nonzero idempotents. Suppose that $R$ contains a pair $e_{1}, e_{2}$ of orthogonal idempotents and that $e_{1} S e_{2} \neq 0$. Then for some $s \in S$ we have $0 \neq a=e_{1} s e_{2}=e_{1} a e_{2}, a^{2}=0$. Since $R$ is a r. P-ideal, we must have $a \in N(R)$, and thus $a R \subseteq N(S)=0$, whereas $0 \neq a e_{2} \in a R$. This contradiction implies that $e_{1} S e_{2}=0$, or $e_{1} S e_{2} S=0$. By the primitivity of $S$ this shows that either $e_{1}=0$ or $e_{2}=0$ (compare [3, Lemma 4]) i.e. $R$ has no pairs of nonzero orthogonal idempotents. By a familiar argument we conclude that for any nonzero idempotent $e$ in $R$ we must have $R=e S$ and that $R$ is minimal. The second part of the theorem follows easily.

ThEOREM 5.6. The P-socle $S^{\prime}$ of a primitive I-ring $S$ coincides with the ordinary socle of $S$.

Proof. This is a consequence of Theorem 5.5 in view of the definition of the $\mathrm{P}$-socle as the sum of all r. P-ideals of the ring.

THEOREM 5.7. If the P-socle of an FI-ring $S$ is not nil, then the ordinary socle of some primitive image of $S$ is not zero.

Proof. By assumption $S$ contains a non nil r. P-ideal $R$. Let $e \neq 0$ be an idempotent in $R$. By Lemma $4.1, e S$ is a r. P-ideal in $S$. The map $\bar{e} \bar{S}$ of $e S$ in some primitive image $\bar{S}$ of $S$ is $\neq 0$. By the corollary to Theorem 5.1 we know that $\bar{e} \bar{S}$ is a r. P-ideal. Hence by Theorem $5.5, \bar{e} \bar{S}$ is a minimal r. ideal in $\bar{S}$, which shows that the socle of $\bar{S}$ is $\neq 0$.

TheOREM 5.8. A ring $S$ is a P-reducible I-ring (FI-ring) if and only if $S$ contains a set $\left\{R_{i}\right\}$ of $r$. ideals with the following properties:

(a) $A=\sum R_{i}$ is an ideal.

(b) $S-A$ is nil.

(c) $R_{i}-N\left(R_{i}\right)$ is plain (resp. strongly regular).

(d) Relation (4) holds for all $R_{i}$.

Proof. Suppose that $S$ is a P-reducible I-ring (FI-ring) and let $\left\{R_{i}\right\}$ constitute the set of all r. P-ideals of $S$. Then assertions (a)-(c) follow by Theorem 2.2, Definition 2.2, and Theorem 5.1 respectively, while (d) holds on account of $S$ being an I-ring. Conversely, if $S$ is a ring with a set of r. ideals $\left\{R_{i}\right\}$ satisfying (a)-(d), then it follows in view of Lemma 3.8 and Theorem 3.3 that $S$ is a P-reducible I-ring (FI-ring).

CoRollary 1. An I-ring (FI-ring) is a P-reducible (FP-reducible) ring if and only if conditions (a), (b), and (c) of Theorem 5.8 are valid.

CoRollary 2. A P-reducible ring is an I-ring (FI-ring) if and only if for every $r . \mathrm{P}$-ideal $R$ condition (4) holds and $R-N(R)$ is plain (strongly regular). 
CoRollary 3. A P-reducible FI-ring is an FP-reducible ring $\left(^{5}\right)$.

We denote by $i(S)$ the maximum of the indices of the nilpotent elements in a ring $S$. If the indices are not bounded, we write $i(S)=\infty$.

THEOREM 5.9. If $R_{1}, R_{2}, \cdots, R_{n}$ are $r$. P-ideals of a semi-simple FI-ring $S$, then the $r$. ideal $A=\sum_{i=1}^{n} R_{i}$ is of bounded index, with bound $i(A)$ satisfying the inequality $i(A) \leqq n+1$. Moreover, every nil-subring $T$ of $A$ is nilpotent and $i(T) \leqq n+1$.

Proof. Consider any primitive image $\bar{S}$ of $S$. Since the r. ideals $R_{i}$ map into the P-socle of $\bar{S}$ (Corollary 3 of Theorem 5.8) it results by Theorem 5.5 that the images of the $R_{i}$ either are zero or are minimal non-nil r. ideals. Thus we may assume that if $\bar{A}$ denotes the image of $A$, then $\bar{A}=\sum_{i=1}^{m} \bar{R}_{i}$, where $m \leqq n$ and the $\bar{R}_{i}$ are minimal non-nil r. ideals. Thus $\bar{A}$ has a left unit $\bar{e}$ and $\bar{A}$ $=\bar{e} \bar{A} \bar{e} \oplus N(\bar{A}), \bar{e} N(\bar{A})=N(\bar{A}), N(\bar{A}) \bar{e}=0$, whereas $\bar{e} \bar{A} \bar{e}$ is isomorphic with a total matrix ring of degree $m$ over a division ring. It is known that every nilsubring of $\bar{e} \bar{A} \bar{e}$ is nilpotent of index $\leqq m$ (compare [8]). Hence every nilsubring of $\bar{A}$ is nilpotent of index $\leqq m+1$. Thus the nil-subring $T$ maps into a nilpotent ring $\bar{T}$ of index $\leqq m+1$. Since this holds for every primitive image of $S$, it follows readily that $T$ itself must be nilpotent of index $\leqq m+1 \leqq n+1$, q.e.d.

Theorem 5.10. $A$ ring $S$ is a P-soluble I-ring (FI-ring) if $S$ possesses a composition chain of ideals $\left\{A_{\sigma}\right\}$ such that $A_{\sigma+1}-A_{\sigma}$ satisfies the following conditions:

(a) There exists a set $\left\{R_{i}^{(\sigma)}\right\}$ of $r$. ideals in $A_{\sigma+1}-A_{\sigma}$ such that $R_{i}^{(\sigma)}-N\left(R_{i}^{(\sigma)}\right)$ is plain (strongly regular).

(b) The sum $B_{\sigma}=\sum_{i} R_{i}^{(\sigma)}$ is an ideal.

(c) The difference ring $\left(A_{\sigma+1}-A_{\sigma}\right)-B_{\sigma}$ is nil.

(d) $N\left(R_{i}^{(\sigma)}\right) \cdot R_{i}^{(\sigma)} \subseteq N\left(A_{\sigma+1}-A_{\sigma}\right)$.

Conversely, every $\mathrm{P}$-soluble FI-ring possesses a composition chain satisfying conditions (a)-(d).

Proof. Suppose that $S$ is a ring possessing a composition chain satisfying (a)-(d). From (b), (c), and (d) it follows by Lemma 3.6 that $N\left(A_{\sigma+1}-A_{\sigma}\right)$ $=N^{*}\left(A_{\sigma+1}-A_{\sigma}\right)$, which, taking into account condition (a), implies in view of Lemma 3.8 and Theorem 3.3 that $A_{\sigma+1}-A_{\sigma}$ is an I-ring (FI-ring). By Theorem 3.2(b) it follows now that $S$ is a P-soluble I-ring (FI-ring). Conversely, suppose that $S$ is a P-soluble FI-ring and let $\left\{A_{\sigma}\right\}$ be a P-chain for $S$. With $S$ also $S-A_{\sigma}$ is an FI-ring and Theorem 5.1 implies that $A_{\sigma+1}-A_{\sigma}$ is a P-reducible FI-ring. By Corollary 2 of Theorem 5.8 it follows therefore that conditions (a)-(d) hold for the set $\left\{R_{i}\right\}$ of all r. P-ideals of $A_{\sigma+1}-A_{\sigma}$.

By Theorem 2.4 and Corollary 3 of Theorem 5.8 we now obtain the important 
Theorem 5.11. Every P-soluble FI-ring $S$ is FP-soluble, that is: Every homomorphic image of $S$ is also a P-soluble FI-ring.

This theorem implies that Theorem 2.5 is available for P-soluble FI-rings and we get

TheOREM 5.12. $A$ ring $S$ is a P-soluble FI-ring if and only if every nonzero $r$. ideal of every homomorphic image of $S$ contains a nonzero $r$. P-ideal $R$ such that $R-N(R)$ is strongly regular and condition (4) holds for $R$. Every P-soluble FI-ring $S$ possesses a uniquely determined P-chain $\left\{B_{\sigma}\right\}$ of "iterated P-socles," i.e. such that $B_{\sigma+1}-B_{\sigma}$ is the $\mathrm{P}$-socle of $S-B_{\sigma}$.

We conclude this section by considering some special cases of $\mathrm{P}$-soluble FI-rings. It will be convenient to introduce the following definition.

Definition 5.2. An element $a$ of a ring $S$ is said to have the finite rank $n$ (notation: $r(a)=n$ ) if the r. ideal $a S$ contains at least one system of $n$ orthogonal idempotents, but no systems of $n+1$ orthogonal idempotents. Otherwise we put $r(a)=\infty$. If no idempotents are available we put $r(a)=0$.

We recall that a ring $S$ is called atomic if it coincides with its ordinary socle, i.e. with the sum of its minimal r. ideals.

Notation. If the descending chain condition on principal non-nil r. ideals holds in a ring $S$, we say that $S$ satisfies the d.c.p. condition.

THEOREM 5.13. If $S$ is a ring with d.c.p. condition then: (1) For every $a \in S$ we have $r(a)<\infty$. (2) The ring $S$ is an I-ring and $S-N(S)$ is atomic. (3) Conversely, (2) implies that the d.c.p. condition holds in $S$.

Proof. If $a S$ is not nil, it contains a primitive idempotent $e_{1}$. Put $a_{1}=a$ $-e_{1} a$. If $a_{1} S$ is not nil, it follows by a well known argument that $a_{1} S$ contains a primitive idempotent $e_{2}$, orthogonal to $e_{1}$. Now $a S \supset a_{1} S$, and by repeating the argument it follows in view of the d.c.p. condition that $a S$ splits into a direct sum of r. ideals, $a S=\sum_{i=1}^{n} e_{i} S+R$, where $R$ is nil and the $e_{i}$ are primitive orthogonal idempotents. Hence $r(a)=n$ and (1) is proved. The proof of (2) and (3) follows readily.

Since for a primitive idempotent $e$ the r. ideal $e S$ is a r. FP-ideal, we have

CoROllary 1. A ring that satisfies the d.c.p. condition is a P-reducible FI-ring.

If $S$ is semi-simple, we obtain the following result, due to I. Kaplansky [6, Theorem 8.1]:

COROLlaRy 2. If a semi-simple ring satisfies the d.c.p. condition, then $S$ is $\operatorname{atomic}\left({ }^{7}\right)$.

If $S$ is an I-ring, $a \in S, r(a)=n$, and $e_{i}, i=1, \cdots, n$, is a system of orthogonal idempotents in $a S$, then it is evident that every $e_{i}$ is a primitive

(7) The present proof is essentially due to 0 . Wyler [12]. 
idempotent. In view of Theorem 5.13 we obtain the following useful characterization of an I-ring that is atomic modulo its radical:

THEOREM 5.14. An I-ring $S$ is atomic modulo its radical if and only if every element of $S$ is of finite rank.

By the above Corollary 1 to Theorem 5.13 it follows that one obtains a special type of $\mathrm{P}$-soluble FI-rings by considering rings that possess a composition chain of ideals $\left\{A_{\sigma}\right\}$ such that every ring $A_{\sigma+1}-A_{\sigma}$ is an I-ring and every element in $A_{\sigma+1}-A_{\sigma}$ is of finite rank. In analogy to Theorem 5.12 one easily obtains the following result.

Theorem 5.15. A ring $S$ possesses a composition chain $\left\{A_{\sigma}\right\}$ such that every ring $A_{\sigma+1}-A_{\sigma}$ is atomic modulo its maximal nil-ideal if and only if every non-nil $r$. ideal in every homomorphic image of $S$ possesses a minimal non-nil r. ideal. [2].

REMARK. The case where $A_{\sigma+1}-A_{\sigma}$ is atomic was considered by R. Baer

6. Conditions for P-solubility of I-rings. The principal aim of the present section is to obtain a complete characterization of P-soluble FI-rings in terms of their primitive images.

Let $e$ be an idempotent in an I-ring $S$ and suppose that $e$ is not a P-idempotent (see Definition 4.1 and Definition 5.1). Then by [10, Theorem 2.1] the I-ring $e S e$ contains a complete matrix units system $e_{i k}^{(1)}, i, k=1, \cdots, m_{1}$, $m_{1} \geqq 2$. If also $e_{11}^{(1)}$ is not a $\mathrm{P}$-idempotent, then the ring $e_{11}^{(1)} S e_{11}^{(1)}$ contains a complete matrix units system $e_{i k}^{(2)}, i, k=1, \cdots, m_{2}, m_{2} \geqq 2$. Thus it follows that in case $S$ is a non-nil I-ring that does not contain P-idempotents, we may continue the process of building this type of complete matrix units systems indefinitely. This leads to the following definitions:

Definition 6.1. A sequence of idempotents

$$
e_{11}^{(1)}, e_{11}^{(2)}, e_{11}^{(3)}, \ldots
$$

is called a decreasing sequence (in short: D-sequence) if the following holds:

(a) The idempotent $e_{11}^{(n)}$ is the first unit in a system of $m_{n}^{2}$ matrix units $e_{i k}^{(n)}, i, k=1, \cdots, m_{n}$, where $m_{n} \geqq 2$.

$$
e_{i k}^{(n)} \in e_{11}^{(n-1)} S e_{11}^{(n-1)}, \quad i, k=1, \cdots, m_{n} ; n=1,2,3, \cdots
$$

Definition 6.2. We say that the D-condition holds in a ring $S$ if every D-sequence in $S$ is finite.

REMARK. The requirement $m_{n} \geqq 2$ implies that the right ideal $e_{11}^{(i)} S$ contains at least two orthogonal idempotents, i.e. $e_{11}^{(i+1)}$ and $e_{22}^{(t+1)}$; hence it follows that $e_{11}^{(i+1)} S$ is a proper subset of $e_{11}^{(i)} S$ and we obtain

Consequence $1^{\circ}$. All terms in a D-sequence (7) are different. 
Consequence 2. The d.c.p. condition implies the D-condition.

We shall presently see that the converse of Consequence 2 is not valid. To this end we assume that the sequence (7) is infinite and start with showing that the ring $e_{11}^{(t-1)} S e_{11}^{(i-1)}$ contains a complete matrix units system of $m_{i}^{2}$ $\cdot m_{t+1}^{2}$ units. Indeed, put $\bar{e}_{s t}^{(i+1)}=\sum_{k=1}^{m_{i}} e_{k 1}^{(i)} e_{s t}^{(i+1)} e_{1 k}^{(i)}$, then each $\bar{e}_{s t}^{(i+1)}$ commutes with each $e_{u v}^{(i)}$ and the set of $m_{i}^{2} \cdot m_{i+1}^{2}$ elements $\bar{e}_{s t}^{(i+1)} e_{u v}^{(i)}, s, t=1, \cdots, m_{i+1}^{2} ; u$, $v=1, \cdots, m_{i}^{2}$ constitutes a complete matrix units system lying in $e_{11}^{(i-1)} S e_{11}^{(i-1)}$. Next note that for the first unit of this system we have $\bar{e}_{11}^{(i+1)} e_{11}^{(i)}=e_{11}^{(i+1)} e_{11}^{(i)}$ $=e_{11}^{(i+1)}$, and since $e_{i k}^{(i+2)} \in e_{11}^{(i+1)} S e_{11}^{(i+1)}$, we may repeat the above procedure and obtain a complete matrix units system of $m_{i}^{2} m_{i+1}^{2} m_{t+2}^{2}$ units lying in $e_{11}^{(i-1)} S e_{11}^{(i-1)}$. Thus proceeding we obtain (by induction) for each $j$ a complete matrix units system of $\prod_{k=1}^{i+j} m_{k}^{2}$ units. Since $m_{k} \geqq 2$, this yields

Lемма 6.1. If (7) is an infinite D-sequence, then the ring $e_{11}^{(n)} S e_{11}^{(n)}$ is of infinite index for every $n$.

COROLlaRY. Every ring with a finite index satisfies the D-condition.

Consider now a strongly regular ring $S$ with unit and with an infinite number of idempotents. Then $S$ is certainly not atomic and thus the d.c.p. condition does not hold in $S$. On the other hand $S$ is of finite index $(=1)$ and thus by the above corollary the D-condition holds in $S$. Hence we have

Consequence 3. The D-condition does not imply the d.c.p. condition even for semi-simple rings.

LEMмA 6.2. In every homomorphism $S \sim \bar{S}$ the nonzero images of the elements of a D-sequence constitute a D-sequence.

Proof. Denote by $\bar{e}_{i k}^{(n)}$ the image of $e_{i k}^{(n)}$. Condition (b) of Definition 6.1 is certainly preserved in $\bar{S}$, while for any $\bar{e}_{11}^{(n)} \neq 0$ it follows by $\bar{e}_{11}^{(n)}=\bar{e}_{11}^{(n)} \bar{e}_{i k}^{(n)} \bar{e}_{k 1}^{(n)}$ that $\bar{e}_{i k} \neq 0, i, k=1,2, \cdots, m_{n}$.

THEOREM 6.1. If the D-condition holds for every primitive image of an I-ring $S$, it holds also for $S$.

Proof. Consider an infinite D-sequence (7) in $S$. In view of condition (b) in Definition 6.1 it follows by a lemma due to Kaplansky (compare [10, Lemma 3.3]) that for some primitive image $\bar{S}$ of $S$ the image $\bar{e}_{11}^{(n)}$ of $e_{11}^{(n)}$ is $\neq 0$ for every $n$, which by Lemma 6.2 (compare also the above Consequence 1 ) is a contradiction. This shows that every $\mathrm{D}$-sequence in $S$ is finite.

ThEOREM 6.2. If the D-condition holds for a non-nil I-ring $S$, then the P-socle $S^{\prime}$ of $S$ is also not nil.

Proof. Without loss of generality we may assume that $S$ is semi-simple. We have to show that $S^{\prime} \neq 0$. By Theorem 5.3 it is sufficient to show that $S$ contains nonzero P-idempotents. Suppose that this is not the case and let $e$ 
be a nonzero idempotent. Then $e S e$ is not plain (Theorem 5.3). By [10, Theorem 2.1] there exists a system of matrix units $e_{i k}^{(1)}, i, k=1, \cdots, m_{1}$, $m_{1} \geqq 2$, so that $e_{i k}^{(1)} \in e S e$. Similarly $e_{11}^{(1)} S e_{11}^{(1)}$ contains a matrix units system $e_{i k}^{(2)}, i$, $k=1, \cdots, m_{2}, m_{2} \geqq 2$, such that $e_{i k}^{(2)} \in e_{11}^{(1)} S e_{11}^{(1)}$. Thus continuing we obtain an infinite D-sequence-a contradiction.

\section{Lemma 6.3. Every P-reducible FI-ring $S$ satisfies the D-condition.}

Proof. We may assume that $S$ is semi-simple. Let $e$ be an idempotent in $S$. It is clear that $e$ lies in the P-socle $S^{\prime}$ of $S$, and thus for a finite set of r. P-ideals $R_{1}, R_{2}, \cdots, R_{n}$ we have $e \in \sum_{i=1}^{n} R_{i}=A$. Then $e S e \subseteq A$. By Theorem 5.9 we know that $A$ and thus also $e S e$ are of bounded index, with upper bound $\leqq n+1$. This shows in view of Lemma 6.1 that every $\mathrm{D}$-sequence in $S$ must be finite.

\section{ThEOREM 6.3. Every P-soluble FI-ring $S$ satisfies the D-condition.}

Proof. We may assume that $S$ is semi-simple. Let $\left\{A_{\sigma}\right\}$ be a P-chain for $S$. Suppose that $S$ contains an infinite D-sequence (7). Start with an arbitrary idempotent $e_{11}^{\left(n_{1}\right)}$ of the sequence and denote by $\sigma_{1}=\sigma_{1}(n)$ the smallest ordinal such that $e_{11}^{\left(n_{1}\right)} \in A_{\sigma_{1}}$. Suppose that $\sigma_{1}=1$, i.e., $e_{11}^{\left(n_{1}\right)}$ lies in the P-reducible ring $A_{1}$. Then $A_{1}$ contains the infinite $\mathrm{D}$-sequence $e_{11}^{\left(n_{1}+r\right)}, r=0,1,2, \cdots$, contradicting Lemma 6.3. Hence $\sigma_{1}>1$. By the minimality of $\sigma_{1}$ it is further clear that $\sigma_{1}$ is not a limit ordinal. Thus we may put $\sigma_{1}=\rho+1$ and we have $e_{11}^{\left(n_{1}\right)}$ $\notin A_{\rho}$. Denote by $\bar{e}_{11}^{(r)}$ the image of $e_{11}^{(r)}$ in the natural homomorphism $S \sim{ }_{\rightarrow} S$ $-A_{\rho}$. Then $\bar{e}_{11}^{\left(n_{1}\right)} \in A_{\rho+1}-A_{\rho}$, which by condition (b) of Definition 6.1 implies that $\bar{e}_{11}^{\left(n_{1}+k\right)} \in A_{\rho+1}-A_{\rho}$ for every $k$. Since $A_{\rho+1}-A_{\rho}$ is a P-reducible FI-ring, it follows by Lemmas 6.2 and 6.3 that for sufficiently large $k$ 's we must have $\bar{e}_{11}^{\left(n_{1}+k\right)}=0$, or $e_{11}^{\left(n_{1}+k\right)} \in A_{\rho}$. Thus we have shown that for some integer $n_{2}>n_{1}$ we have $e_{11}^{\left(n_{2}\right)} \in A_{\rho}$. Choose the smallest integer of this kind and denote by $\sigma_{2}=\sigma_{2}(n)$ the smallest ordinal such that $e_{11}^{\left(n_{2}\right)} \in A_{\sigma_{2}}$, then $\sigma_{2}<\sigma_{1}$. This process leads to an infinite decreasing sequence of ordinals $\sigma_{1}>\sigma_{2}>\ldots$ - contradiction which shows that there are no infinite $\mathrm{D}$-sequences in $S$, q.e.d.

THEOREM 6.4. An FI-ring $S$ is $\mathrm{P}$-soluble if and only if the $\mathrm{D}$-condition holds for every primitive image of $S$.

Proof. Sufficiency. Denote by $A_{1}$ the P-socle of $S$. We have remarked already that the P-socle of an I-ring $S$ contains the radical $N(S)$ of $S$. By Theorem 6.2 it follows now that whenever $S \supset 0$ also $A_{1} \supset 0$. If $A_{1}=S$ the theorem is proved. If not, consider $S-A_{1}$. With $S$ also $S-A_{1}$ is an FI-ring whose primitive images are primitive images of $S$, thus satisfying the D-condition. Hence the procedure may be repeated and in familiar manner leads to a $\mathrm{P}$-chain for $S$, i.e. $S$ is $\mathrm{P}$-soluble.

Necessity. Let now $S$ be a P-soluble FI-ring. Then also every primitive 
image $\bar{S}$ of $S$ is a P-soluble FI-ring (Theorem 5.11). Hence the D-condition holds in $\bar{S}$ (Theorem 6.3).

Theorem 6.4 and Theorem 5.11 lead to the following

Corollary. An FI-ring $S$ is $\mathrm{P}$-soluble if and only if every primitive image of $S$ is P-soluble.

We have seen (Theorem 6.1) that the $\mathrm{D}$-condition for the primitive images of an I-ring implies its validity for the ring itself. The question presents itself whether for an FI-ring the converse is also true. We are unable to settle the problem generally, but we shall answer it in the affirmative for algebraic algebras. We shall see that in this case the $\mathrm{D}$-condition is preserved under every homomorphism. For the remaining part of this section let $S$ denote an algebraic algebra over a field $\phi$, and $A$ an ideal in $S$. Write $b \equiv c$ if $b-c \in A$.

Lemma $6.4\left({ }^{8}\right)$. For any element $e$ which is idempotent modulo $A$ there exists an idempotent $d$ in $S$ such that $d \equiv e, d$ a polynomial( $\left.{ }^{9}\right)$ in $e$.

Proof. By assumption $e^{2}=e+a, a \in A$. Denote by $e^{\prime}$ the principal idempotent of the algebra $(a)_{\phi}$ generated by $a$ over $\phi$. Then $b=a-e^{\prime} a$ is a nilpotent element of $A$. Put $d^{\prime}=e-e^{\prime} e$. Since the elements $a, e^{\prime}$ and $b$ are polynomials in $e$, they permute with each other and with $e$, and one finds readily that $d^{\prime 2}=d^{\prime}+b, d^{\prime} \equiv e$. Since $b$ is nilpotent, it follows in a familiar manner that there is an idempotent $d$ such that $d \equiv d^{\prime}$ and $d$ a polynomial in $d^{\prime}$. Since with $d^{\prime}$ also $d$ is a polynomial in $e$, the proof of the lemma is complete.

Lemma 6.5. Let $e_{1}, e_{2}$ be a pair of orthogonal idempotents modulo A. Then there exists a pair of orthogonal idempotents $d_{1}, d_{2}$ such that $d_{i}=e_{i}, i=1,2$.

Proof. By Lemma 6.4 there exists an idempotent $d_{1}$ such that $d_{1} \equiv e_{1}$, $d_{1} e_{1}=e_{1} d_{1}$. Put $d_{2}^{\prime}=e_{2}-d_{1} e_{2}-e_{2} d_{1}+d_{1} e_{2} d_{1}$, then $d_{1} d_{2}^{\prime}=d_{2}^{\prime} d_{1}=0$. Since $d_{2}^{\prime} \equiv e_{2}$ we have $d_{2}^{\prime 2} \equiv e_{2}^{2} \equiv e_{2} \equiv d_{2}^{\prime}$ and thus, again by Lemma 6.4 , we can find an idempotent $d_{2}$, a polynomial in $d_{2}^{\prime}$ such that $d_{2} \equiv d_{2}^{\prime} \equiv e_{2}$. Since $d_{1} d_{2}^{\prime}=d_{2}^{\prime} d_{1}=0$ and $d_{2}$ is a polynomial in $d_{2}^{\prime}$, it follows that $d_{1} d_{2}=d_{2} d_{1}=0$, which completes the proof.

Lemma 6.6. Let $e_{i}, i=1, \cdots, n$, be a set of orthogonal idempotents modulo $A$. Then there exists in $S$ a system of orthogonal idempotents, $d_{i}, i=1, \cdots, n$, such that $d_{i} \equiv e_{i}$.

Proof. We use induction. By Lemmas 6.4 and 6.5 we may assume that $n \geqq 3$. Put $e^{(2)}=\sum_{i=2}^{n} e_{i}$. The pair $e_{1}, e^{(2)}$ is orthogonal modulo $A$, hence by Lemma 6.5 there is a pair of orthogonal idempotents $d_{1}, d^{(2)}$ such that

(8) The author is indebted to Professor N. Jacobson from whom he has learned about the validity of this lemma, and who also conjectured Theorem 6.5 .

(9) All occurring polynomials are such over the field $\phi$, with constant coefficient $=0$. 
$d_{1} \equiv e_{1}, d^{(2)} \equiv e^{(2)}$. Consider the algebra $S^{\prime}=d^{(2)} S d^{(2)}$ and the ideal $A^{\prime}=d^{(2)} A d^{(2)}$ in $S^{\prime}$. Then $A^{\prime} \subseteq A$ and the elements $d_{i}^{\prime}=d^{(2)} e_{i} d^{(2)}, i=1, \cdots, n$, constitute an orthogonal system modulo $A^{\prime}$ (and hence also modulo $A$ ). By induction there exists a system of $n-1$ orthogonal idempotents $d_{i}, i=2, \cdots, n$, such that $d_{i} \equiv d_{i}^{\prime}, i=2, \cdots, n$. Since $d_{i}^{\prime} \equiv e_{i}$, we have also $d_{i} \equiv e_{i}$. By $d_{1} d^{(2)}$ $=d^{(2)} d_{1}=0$ and $d_{i} \in d^{(2)} S d^{(2)}, i \geqq 2$, it follows that the system $d_{i}, i=1, \cdots, n$, satisfies the requirements of the lemma.

THEOREM 6.5. Let $e_{i k}$ be a system of $n^{2}$ matrix units modulo $A$. Then there exists in $S$ a system of $n^{2}$ matrix units $d_{i k}$ such that $d_{i k} \equiv e_{i k}$.

Proof. By Lemma 6.6 there is in $S$ an orthogonal system of idempotents $e_{i i}^{\prime}, i=1, \cdots, n$, such that $e_{i j}^{\prime} \equiv e_{i i}$. Put $d_{i j}^{\prime}=e_{i t}^{\prime} e_{i j} e_{j j}^{\prime}$, then $d_{i j}^{\prime} \equiv e_{i j}^{\prime}$ and we have $d_{i j}^{\prime} d_{j^{\prime} k}^{\prime}=0$ for $j \neq j^{\prime}$. Consider the elements $a=\sum_{i=1}^{n-1} d_{i t+1}^{\prime}, b=\sum_{i=1}^{n-1} d_{t+1}^{\prime}$, and $c=a^{n-1} b^{n-1}$. Evidently $c \equiv d_{11}^{\prime}$. Hence $c$ is idempotent modulo $A$ and thus by Lemma 6.4 there is an idempotent $d_{11}$, a polynomial in $c$ such that $d_{11}$ $\equiv d_{11}^{\prime} \equiv c$. For some polynomial $g(c)$ we have $d_{11}=c g(c)=a^{n-1} b^{n-1} g(c)$. Put $q$ $=b^{n-1} g(c) d_{11}$. Then $d_{11}=a^{n-1} q$ and it follows readily by $c \cdot d_{11}^{\prime}=d_{11}^{\prime} c=c$ that the elements $d_{i i}=a^{n-i} q a^{i-1}, i=1, \cdots, n$, constitute a system of orthogonal idempotents, whereas the elements $d_{i k}=a^{n-1} q a^{k-1}, i, k=1, \cdots, n$, form a system of $n^{2}$ matrix units. Now note that by $d_{11} \equiv c, d_{11}=c g(c)$ it follows that $g(c) \equiv d_{11}$. This implies that $d_{i k} \equiv e_{i k}$, which completes the proof of the theorem.

THEOREM 6.6. If the $\mathrm{D}$-condition holds in an algebraic algebra $S$, it holds also in every homomorphic image $T$ of $S$.

Proof. Suppose that $T$ contains an infinite D-sequence (7). By Theorem 6.5 there is in $S$ a system of matrix units $d_{i k}^{(1)}, i, k=1, \cdots, m_{1}, m_{1} \geqq 2$, such that $d_{i k}^{(1)}$ maps into $e_{i k}^{(1)}$ under the homomorphism $S \sim \rightarrow T$. Consider the induced homomorphism

$$
d_{11}^{(1)} S d_{11}^{(1)} \sim_{+} e_{11}^{(1)} T e_{11}^{(1)} .
$$

Since $e_{i k}^{(2)} \in e_{11}^{(1)} T e_{11}^{(1)}$, we can find (again by Theorem 6.5) in $d_{11}^{(1)} S d_{11}^{(1)}$ a system of matrix units $d_{i k}^{(2)}, i, k=1, \cdots, m_{2}, m_{2} \geqq 2$, such that $d_{i k}^{(2)}$ maps into $e_{i k}^{(2)}$ under the homomorphism (8). Thus we obtain an infinite D-sequence $d_{11}^{(n)}$, $n=1,2,3, \cdots$, in $T$ - a contradiction.

In view of Theorem 6.1 we have the following

CoROLLARY. The D-condition holds in an algebraic algebra $S$ if and only if it holds in every primitive image of $S$.

Theorems 6.1, 6.4 and 6.6 now yield

THEOREM 6.7. An algebraic algebra is P-soluble if and only if it satisfies the D-condition.

REMARK. This is also a consequence of Theorems 6.3 and 6.6. 
7. On subrings of P-soluble FI-rings. In the present section some properties of FI-subrings of a P-soluble FI-ring are derived and in particular a result due to $R$. Baer is sharpened and generalized.

We have seen (Theorem 6.5) that if a system of $n^{2}$ elements $e_{i k}, i, k$ $=1, \cdots, n$, in an algebraic algebra forms a matrix units system modulo an ideal $A$, then there is a matrix units system $d_{i k}$ in the algebra such that $d_{i k}$ $-e_{i k} \in A$. It is readily verified that this theorem remains valid for any ring, provided that the ideal $A$ is nil. This leads in view of [10, Theorem 3.3] to the following

LemMa 7.1. If $R$ is a r. I-ideal in an arbitrary ring $S$ and $R-N(R)$ is of bounded index with upper bound $i(R-N(R))=n$, then $R$ contains a system of $n^{2}$ matrix units $e_{i k}, i, k=1, \cdots, n$. The $e_{i i}$ are P-idempotents of $S$ and thus (Theorem 5.3) the $e_{i i} S$ are r. P-ideals of $S$.

COROLlaRy. If $R$ is a non-nil r. I-ideal in a ring $S$ and $R-N(R)$ is of bounded index, then the crosscut of $R$ with the $\mathrm{P}$-socle of $S$ is not nil.

Lemma 7.2. Let $R$ be a non-nil $r$. FI-ideal of a ring $S$ and $S^{\prime}$ the P-socle of $S$. Suppose that $i(R-N(R))<\infty$ and put $R_{1}=\left(R+S^{\prime}\right)-S^{\prime}$. Then

$$
i\left(R_{1}-N\left(R_{1}\right)\right)<i(R-N(R)) .
$$

Proof. Define the ideal $R^{*}$ of $R$ such that $R^{*} \supseteq N(R)$ and $R^{*}-N(R)$ is the sum of all matrix ideals of $R-N(R)=\bar{R}$; then by [10, Lemma 5.1] we have

$$
i(R-N(R))>i\left(R-R^{*}\right) .
$$

Let $A$ be a matrix ideal of $R$ of degree $n$ modulo $N(R)$, and let $e$ be an idempotent in $R$ which is the identity of $A$ modulo $N(R)$. Since $A$ is a sum of $n$ r. P-ideals $\left({ }^{6}\right)$ modulo $N(R)$, it follows that $e \in S^{\prime}$. Thus the ideal (e) generated by $e$ in $R$ is a subset of $S^{\prime}$ and we have $(e)+N(R) \subseteq S^{\prime} \cap R+N(R)$; consequently $R^{*} \subseteq R^{* *}=S^{\prime} \cap R+N(R)$. Now $R^{*}-R \cap S^{\prime} \subseteq R^{* *}-R \cap S^{\prime} \subseteq N(R-R$ $\left.\cap S^{\prime}\right)$ and by applying $\left[10\right.$, Theorem 5.4] we get $i\left(R_{1}-N\left(R_{1}\right)\right)=i\left(\left(R-R \cap S^{\prime}\right)\right.$ $\left.-N\left(R-R \cap S^{\prime}\right)\right) \leqq i\left(\left(R-R \cap S^{\prime}\right)-\left(R^{* *}-R \cap S^{\prime}\right)\right) \leqq i\left(\left(R-R \cap S^{\prime}\right)-\left(R^{*}-R\right.\right.$ $\left.\left.\cap S^{\prime}\right)\right)=i\left(R-R^{*}\right)$, or $i\left(R_{1}-N\left(R_{1}\right)\right) \leqq i\left(R-R^{*}\right)$, which in view of (10) yields the required inequality (9).

Lemma 7.3. Let $S$ be a P-soluble FI-ring and $\left\{A_{\sigma}\right\}$ the P-chain of iterated P-socles (compare Theorem 5.12). Suppose that $R$ is a r. ideal of $S$ such that $n=i(R-N(R))<\infty$. Then for some finite integer $m \leqq n$ we have $R \leqq A_{\sigma}$ if $\sigma \geqq m+1$.

Proof. Put $B_{k}=\left(R+A_{k}\right)-A_{k}$. If $B_{k}=N\left(B_{k}\right)$, then in view of the fact that the P-socle contains all nil-ideals, we have $R \subseteq A_{k+1}$. If $B_{k} \supset N\left(B_{k}\right)$, then by Lemma 7.2 one readily obtains

$$
i\left(B_{k}-N\left(B_{k}\right)\right)<i\left(B_{k+1}-N\left(B_{k+1}\right)\right) .
$$


Since with $B_{k}$ also $B_{j}, j<k$, is not nil, we obtain by (11)

$$
n=i\left(B_{0}-N\left(B_{0}\right)\right)>i\left(B_{1}-N\left(B_{1}\right)\right)>\cdots>i\left(B_{k}-N\left(B_{k}\right)\right)
$$

which shows that for some $m \leqq n$ the r. ideal $B_{m}$ must be nil and thus $B_{m}$ $\subseteq A_{m+1}-A_{m}$, or $R \subseteq A_{m+1}$, q.e.d.

LEMMA 7.4. Suppose that the FI-ring $S$ is the sum of all $r$. ideals of a set $\left\{R_{i}\right\}$ such that $i\left(R_{i}-N\left(R_{i}\right)\right)<\infty$ for every $i$, and let $\left\{A_{\sigma}\right\}$ be the P-chain of iterated $\mathrm{P}$-socles in $S$. Then $S$ is $\mathrm{P}$-soluble and $S=A_{\omega}$.

Proof. Every element $a$ of $S$ lies in the sum $R$ of a finite number of the $R_{i}$ and for this sum an integer $m$ may be fixed (Lemma 7.3) so that $R \subseteq A_{m}$, or $a \in A_{m}$. Hence $S \subseteq \mathrm{U}_{\sigma<\omega} A_{\sigma}$, which shows that $S=A_{\omega}$.

REMARK. For the "actual" length of the P-chain $A_{\sigma}$, i.e. for the smallest ordinal $\tau$ such that $A_{\tau}=A_{\tau+1}$, we may have $\tau<\omega$.

TheOREM 7.1. Every FI-subring $T$ of a P-reducible FI-ring $S$ is P-soluble. If $\left\{B_{\sigma}\right\}$ is the $\mathrm{P}$-chain of iterated $\mathrm{P}$-socles of $T$, then $T=B_{\omega+1}$. If $S$ coincides with its $\mathrm{P}$-socle, then $T=B_{\omega}$.

Proof. Let $\left\{R_{i}\right\}$ be the set of r. P-ideals of $S$, so that $S^{\prime}=\sum R_{i}$ is the P-socle of $S$ and $S-S^{\prime}$ is nil. Suppose first that $S=S^{\prime}$. In this case every element $t \in T$ lies in the sum of a finite number of the $R_{i}$ so that for some integer $m$ we have $t \in R=\sum_{i=1}^{m} R_{i}$. By Theorem 5.9 we know that $i[(R+N(S))$ $-N(S)] \leqq m+1$. This yields for the r. ideal $(t)_{r}$ generated by $t$ in $T$ the inequality $\left(^{10}\right) i\left[(t)_{r}-N\left((t)_{r}\right)\right] \leqq m+1$. Since every ring is the sum of its principal r. ideals, it follows by Lemma 7.4 that $T=B_{\omega}$. In case $S \supset S^{\prime}$, we know already at any rate that $T \cap S^{\prime}$ is P-soluble and has a $\mathrm{P}$-chain $\left\{C_{\sigma}\right\}$ of iterated P-socles having the length $\omega$. Since $S-S^{\prime}$ is nil and $T-\left(T \cap S^{\prime}\right) \cong\left(T+S^{\prime}\right)$ $-S^{\prime}$, also $T-\left(T \cap S^{\prime}\right)$ is nil and thus coincides with its $\mathrm{P}$-socle $T-C_{\omega}$. Thus by putting $T=C_{\omega+1}$, we see that the $\left\{C_{\sigma}\right\}$ form the required P-chain for $T$.

TheOREM 7.2. Every FI-subring $T$ of a P-soluble FI-ring $S$ is $\mathrm{P}$-soluble.

Proof. Consider the P-chain $\left\{A_{\sigma}\right\}$ for $S$. By Lemma 2.2 we know that the ideals $A_{\sigma} \cap T$ constitute a composition chain for $T$, where for every $\sigma$ the ring $T_{\sigma}=\left(A_{\sigma+1} \cap T\right)-\left(A_{\sigma} \cap T\right)$ is isomorphic with a subring of the P-reducible FI-ring $A_{\sigma+1}-A_{\sigma}$. Since by Theorem 7.1 all $T_{\sigma}$ are $\mathrm{P}$-soluble, it follows readily by transfinite induction that $T$ itself is also $\mathrm{P}$-soluble.

CoRollary. Every subalgebra of a $\mathrm{P}$-soluble algebraic algebra is $\mathrm{P}$-soluble.

REMARK. The above corollary is also an easy consequence of Theorem 6.7, since it is clear that if the D-condition holds in a ring $S$, it holds also in every

(10) Indeed, $i\left[(t)_{r}-N\left((t)_{r}\right)\right] \leqq i\left((t)_{r}-N(S) \bigcap(t)_{r}\right)=i\left[\left((t)_{r}+N(S)\right)-N(S)\right] \leqq i((R+N(S)$ $-N(S)$ ). 
subring of $S$. We consider now some special cases and first derive a simple proof for the following theorem, due to A. Rosenberg [11, Theorem 1].

THEOREM 7.3. Let $S$ be atomic modulo its maximal nil-ideal. Then every I-subring $T$ of $S$ is also atomic modulo its radical.

Proof. For $a \in T$ denote by $r(a)$ the rank of $a$ in $S$ (see Definition 5.2) and by $r^{\prime}(a)$ the rank of $a$ in $T$. Evidently $r^{\prime}(a) \leqq r(a)$. By Theorem 5.14 we know that $r(a)<\infty$, hence $r^{\prime}(a)<\infty$, which implies (Theorem 5.14) that $T$ is atomic modulo its radical.

By Theorems 5.15 and 7.3 we obtain, by an argument similar to that used in the proof of Theorem 7.2, the following result:

THEOREM 7.4. Let $S$ be a ring with the property: Every non-nil $r$. ideal in every homomorphic image of $S$ contains a minimal $r$. ideal. Then every I-subring of $S$ has the same property.

Turning now in particular to the study of the nil-subrings of a $\mathrm{P}$-soluble FI-ring, we recall first that an ordinary radical of any ring $S$ is defined as the sum of all nilpotent ideals of $S$, and that a composition chain $\left\{N_{\sigma}\right\}$ of iterated ordinary radicals may be formed in $S$ (where $N_{\sigma+1}-N_{\sigma}$ is the ordinary radical of $S-N_{\sigma}$ ), terminating, say, at $N_{\tau}=L(S)$. The ideal $L(S)$ is called the lower radical of $S$ (see [2]). The ring $S-L(S)$ has no nonzero nilpotent ideals, whereas for any nonzero subring of $L(S)$ the opposite is true. A ring $S$ may be termed an $L$-ring if $\left.S=L(S){ }^{11}\right)$. We also recall that every $L$-ring is seminilpotent, i.e. every finite set in an L-ring generates a nilpotent ring, whereas there exist semi-nilpotent rings that are not L-rings. We shall see that the nil-subrings of a P-soluble FI-ring are closely connected with L-rings.

One proves readily

Lemma 7.5. If a ring $S$ has a composition chain $\left\{A_{\sigma}\right\}$ of ideals such that $A_{\sigma+1}-A_{\sigma}$ is an L-ring, then also $S$ is an L-ring.

We prove now

THEOREM 7.5. If a semi-simple P-reducible FI-ring $S$ coincides with its $\mathrm{P}$-socle, then every nil-subring $T$ of $S$ coincides with its ordinary radical, i.e. $T$ is a special L-ring.

Proof. In this case $S=\sum R_{i}$, where $\left\{R_{i}\right\}$ is the set of r. P-ideals of $S$. The nil-r. ideal $(t)_{r}$ generated by an element $t \in T$ in $T$ lies in a sum of a finite set of $R$ 's and is therefore by Theorem 5.9 a nilpotent $\mathrm{r}$. ideal of $T$. Thus $T$ is the sum of its nilpotent r. ideals, q.e.d.

In view of Lemma 7.5 we get the following

Corollary 1. Let $S$ be a P-reducible FI-ring and $S^{\prime}$ its P-socle. If the nilrings $N(S)$ and $S-S^{\prime}$ are L-rings, then every nil-subring of $S$ is an L-ring.

(11) An $L$-ring is evidently an FE-soluble ring, with nilpotence as the underlying $\mathbb{E}$-property 
Since every homomorphic image of a regular ring is semi-simple, we get

CoROLlaRy 2. Every nil-subring of a P-reducible regular ring is an L-ring that coincides with its ordinary radical.

Theorem 7.6. Let $S$ be a P-soluble FI-ring and let $\left\{B_{\sigma}\right\}$ be its P-chain of iterated $\mathrm{P}$-socles. If $N\left(B_{\sigma+1}-B_{\sigma}\right)$ is an L-ring for every $\sigma$, then every nil-subring $T$ of $S$ is an L-ring.

Proof. Consider the composition chain $\left\{C_{\sigma}\right\}$ for $T$ where $C_{\sigma}=T \cap B_{\sigma}$ (see Lemma 2.2). Since $C_{\sigma+1}-C_{\sigma}$ is isomorphic with a subring of $B_{\sigma+1}-B_{\sigma}$ we know by Theorem 7.5 that $C_{\sigma+1}-C_{\sigma}$ is an L-ring. Hence by Lemma 7.5 it follows that $T$ is an L-ring.

In particular we have

COROLLARY 1. The radical of a ring satisfying the conditions of Theorem 7.6 coincides with its lower radical.

REMARK. This corollary is a generalization of a theorem due to R. Baer (see $\left[2\right.$, Theorem 5.1]) concerning rings with a composition chain $\left\{A_{\sigma}\right\}$ of iterated socles (i.e. $A_{\sigma+1}-A_{\sigma}$ is the sum of all minimal r. ideals of $\left.S-A_{\sigma}\right)$. In this case we have $\left(N\left(A_{\sigma+1}-A_{\sigma}\right)\right)^{2}=0$, i.e. $N\left(A_{\sigma+1}-A_{\sigma}\right)$ is a very special L-ring.

For regular rings we evidently get

COROLlary 2. Every nil-subring of a P-soluble regular ring is an L-ring.

Finally we state

COROLlaRY 3. If the radical of every homomorphic image of a P-soluble FIring is semi-nilpotent, then every nil-subring of $S$ is semi-nilpotent.

8. Kurosch's problem. Let $S$ denote an algebraic algebra over a field $\phi$. Kurosch's problem (compare [4]) concerning the local finiteness of $S$ has been settled up to now under the following three conditions (compare [6], [7], and [10]):

Condition 8.1. The minimal condition on r. ideals holds in every primitive image of $S$.

Condition 8.2. If $\Phi$ is the division algebra of endomorphisms of the minimal r. ideals of a primitive image $\bar{S}$ of $S$, then $\bar{\phi}$ is of finite dimension over its center.

Condition 8.3. The radical of every homomorphic image of $S$ is locally finite.

REMARK. By a theorem due to I. Kaplansky [5], conditions 8.1 and 8.2 taken together are equivalent to the single condition: Every primitive image of $S$ satisfies a polynomial identity. We further note that all three conditions hold if the algebra $S$ satisfies a polynomial identity.

The aim of the present section is to show that condition 8.1 can be considerably slackened. 
LEMMA 8.1. If the locally finite kernel( $\left.{ }^{12}\right) K(S)$ of an algebra $S$ is $=0$, and if $e$ is a nonzero idempotent in $S$, then the algebra eSe is not locally finite.

Proof. Suppose that $e S e$ is locally finite and consider the r. ideal $R=e S$. Then $R=e S e \oplus N, N$ a nilpotent ideal in $R$, i.e. locally finite. Now with $N$ and $e S e \cong R-N$ also $R$ must be locally finite, contradicting the assumption that $K(S)=0$.

THEOREM 8.1. If an algebra $S$ satisfies the D-condition for every primitive image $\left({ }^{13}\right)$ of $S$, as well as conditions 8.2 and 8.3 , then $S$ is locally finite.

Proof. Denote by $K(S)$ the locally finite kernel of $S$. Condition 8.3 implies that $T=S-K(S)$ is semi-simple. At any rate the locally finite kernel $K(T)$ of $T$ is $=0$ (see [10, Theorem 6.3]). Suppose that $T \neq 0$. Since the D-condition holds for the primitive images of $T$ it follows by Theorem 6.4 that $T$ is $\mathrm{P}$-soluble, and thus the P-socle $T^{\prime}$ of $T$ is $\neq 0$. Since $\left({ }^{14}\right) N(T)=0$, it follows that $T^{\prime}$ is not nil and thus contains a nonzero P-idempotent $e$. The algebra $e T e$ is strongly regular. The primitive images of $e T e$ are those $\neq 0$ images that are induced by the primitive images of $T$ (see [6, Theorem 3.1]). If now $\bar{e}$ is the image of $e$ in the homomorphism $T \sim \vec{T}$, where $\bar{T}$ is primitive, then $\bar{e} \bar{T} \bar{e}$ is a division algebra $\left.{ }^{15}\right)$ and thus isomorphic with the division algebra of endomorphisms of the minimal r. ideals of $\bar{T}$. By condition 8.2 we know that $\bar{e} \bar{T} \bar{e}$ is locally finite. Hence conditions $8.1,8.2$, and 8.3 hold for $e T e$, i.e. this algebra is locally finite. By Lemma 8.1 it follows now that $e T$ is locally finite, i.e. $0 \neq e T \subseteq K(T)=0$-a contradiction which shows that we must have $T=0$, or $S=K(S)$, q.e.d.

\section{BIBLIOGRAPHY}

1. A. S. Amitsur, A general theory of radicals, Amer. J. Math. vol. 74 (1952) pp. 774-786.

2. R. Baer, Radical ideals, Amer. J. Math. vol. 65 (1943) pp. 537-568.

3. N. Jacobson, The radical and semi-simplicity for arbitrary rings, Amer. J. Math. vol. 67 (1945) pp. 300-320.

4. - Structure theory for algebraic algebras of bounded degree, Ann. of Math. vol. 46 (1945) pp. 695-707.

5. I. Kaplansky, Rings with a polynomial identity, Bull. Amer. Math. Soc. vol. 54 (1948) pp. 575-580.

6. - Topological representation of algebras, Trans. Amer. Math. Soc. vol. 68 (1950) pp. $62-75$.

7. - The structure of certain operator algebras, Trans. Amer. Math. Soc. vol. 70 (1951) pp. 219-255.

(12) For the definition and properties of $K(S)$ compare [10].

(13) By the corollary to Theorem 6.6 this is equivalent to assuming the D-condition for the algebra $S$.

(14) This is a consequence of condition 8.3 and [10, Theorem 6.3].

(15) With $\bar{T}$ also $\bar{e} \bar{T} \bar{e}$ is primitive. On the other hand this algebra is strongly regular, being a homomorphic image of the strongly regular algebra $e T e$. This implies that $\bar{e} \bar{T} \bar{e}$ must be a division algebra. 
8. J. Levitzki, Ueber nilpotente Unterringe, Math. Ann. vol. 105 (1931) pp. 620-627.

9. - A theorem on polynomial identities, Proc. Amer. Math. Soc. vol. 1 (1950) pp. 334-341.

10. - On the structure of algebraic algebras and related rings, Trans. Amer. Math. Soc. vol. 74 (1953) pp. 384-409.

11. A. Rosenberg, Subrings of simple rings with minimal ideals, Trans. Amer. Math. Soc. vol. 73 (1952) pp. 115-138.

12. O. Wyler, Ueber einen Rangbegriff in der Theorie der Ringe speziell der regulären Ringe, Compositio Math. vol. 9 (1951) pp. 193-208.

HEBREW UNIVERSITY, JERUSALEM, ISRAEL. 